\title{
Polymathematics : is mathematics a single science or a set of arts?
}

\author{
V.I. Arnold \\ Steklov Mathematics Institute, Moscow \\ CEREMADE, Université Paris-Dauphine *†
}

The shaft becomes too deep ... and if new veins would not be discovered, places for geometry in the Academy will become what already are the Arabic chairs at the Universities.

Lagrange to D'Alembert, 1781

All mathematics is divided into three parts: cryptography (paid for by CIA, KGB and the like), hydrodynamics (supported by manufacturers of atomic submarines) and celestial mechanics (financed by military and by other institutions dealing with missiles, such as NASA.).

Cryptography has generated number theory, algebraic geometry over finite fields, algebra ${ }^{1}$, combinatorics and computers.

Hydrodynamics procreated complex analysis, partial derivative equations, Lie groups and algebra theory, cohomology theory and scientific computing.

Celestial mechanics is the origin of dynamical systems, linear algebra, topology, variational calculus and symplectic geometry.

The existence of mysterious relations between all these different domains is the most striking and delightful feature of mathematics (having no rational explanation).

The experience of the past centuries shows that the development of mathematics was due not to technical progress (consuming most of the efforts of mathematicians at any given moment), but rather to discoveries of unexpected interrelations between different domains (which were made possible by these efforts).

${ }^{*}$ Partially supported by the Russian Basic Research Foundation, project 99-01-01109, and by the Institut Universitaire de France.

${ }^{\dagger}$ The author thanks I. Mazella and J.-O. Moussafir for the typing help.

${ }^{1}$ The creator of modern algebra, Viète, was the cryptographer of King Henry IV of France. 
The precious technical reports of the present state of different mathematical domains reminds us of the trench warfare. The description of the front line with its bends and everyday moves is of course highly important for the battle participants.

But the pernicious character of the separating tendencies (to which the growing specialisation of mathematicians and the fragmentation of mathematics into small domains leads) becomes evident when one tries to understand the development of mathematics in the past with all its meanders.

Sylvester(1876) already described as an astonishing intellectual phenomenon, the fact that general statements are simpler than their particular cases. The antibourbakist conclusion that he drew from this observation is even more striking. According to Sylvester, a mathematical idea should not be petrified in a formalised axiomatic setting, but should be considered instead as flowing as a river. One should always be ready to change the axioms, preserving the informal idea.

Consider for instance the idea of a number. It is impossible to discover quaternions trying to generalise real, rational, complex or algebraic number fields.

The possibility of such informal generalisation of all mathematics, for which we have no ready axioms, seems to me the most appealing dream.

The informal complexification, quaternionization, symplectization, contactization etc., described below, are acting not on such small things, as points, functions, varieties, categories or functors, but on the whole of mathematics.

I have successfully used these ideas many times as a method to guess new results. I hope therefore that in the future this method of the multiplication of mathematics will be as standard, as is now the transition from finite-dimensional linear algebra to the theory of integral equations and to functional analysis.

Perhaps, the simplest example of this multiplication paradigm is provided by the Killing-Coxeter theory of the reflection groups. Linear algebra is essentially the theory of the special roots systems $A_{k}$. The basic facts of linear algebra (like the eigenvalues and the Jordan blocks theory) can be reformulated in terms of the roots, making the statements meaningful for other systems of roots. These new statements miraculously occur to be correct (while suitably modified). The theories of the roots systems $B_{k}, C_{k}, D_{k}$ (corresponding to the Euclidean and symplectic spaces geometry) are from this point of view rather the sisters than the daughters of the usual vector space geometry (even though they are, of course, the geometries of the usual vector spaces endowed with additional structures).

By the way, many of the results of these theories are in fact also true not only for the exceptional crystallographic groups $\left(E_{6}, E_{7}, E_{8}, F_{4}, G_{2}\right)$ but also for the noncrystallographic Euclidean reflection groups $\left(I_{2}(p), H_{3}, H_{4}\right)$. For example, starting from the ideas of O. Scherbak (1985), A.B. Givental (1988) discovered in the theory of Lagrange and Legendre projections in symplectic and contact geometry a geometrical problem, whose solutions are in a natural one-to-one correspondence with 
the Euclidean reflection groups (and not just with the crystallographic ones as in my preceding theory). This is the problem of classification of the simple projections (having no continuous invariants) of the (virtually singular) Lagrange and Legendre subvarieties.

The main applications of the mathematics multiplication idea are not to be found in finite dimensional algebra but rather in infinite-dimensional calculus, where the Killing classification of the simple Lie algebras is replaced by the Cartan classification of the simple Lie pseudo-groups.

For example the symplectization idea suggests that all differential geometry and topology notions and results should have symplectic versions - the symplectic geometry and the symplectic topology.

In some cases these versions are quite obvious. The ordinary vector fields correspond to the Hamiltonian ones, their Poisson brackets algebra to the Poisson brackets of the Hamilton functions, the strings should move from the configuration space to the phase space and so on. In other cases this generalisation is less evident. For instance, the submanifolds of the ordinary geometry correspond to the Lagrangian submanifolds of symplectic manifolds (A. Weinstein's Principle).

Trying to symplectize the Euler-Poincare theorem on the sum of the indices of the singular points of vector fields, I was lead in 1965 to the conclusion that the symplectization should be an astonishing extension of the Morse theory. I formulated "Arnold's conjectures" on the fixed points of symplectomorphisms, on the intersections of the Lagrangian submanifolds and on the linkings of the Legendrian ones.

The simplest of these conjectures states that the number of fixed points of an area preserving diffeomorphism of a two-torus to itself, "preserving the mass centre", has at least 4 fixed points (taking the multiplicities into account).

In the more general case of a compact symplectic manifold, the diffeomorphism should be the time one map of a flow defined by time-dependent Hamilton function, and the number of fixed points is minorated by the minimal number of the critical points of a function on the manifold (the fixed and critical points being counted either both with their multiplicities, or in both cases geometrically).

These conjectures, generalising the "last theorem" of Poincaré on the circular annulus mapping, have been later studied in a series of brilliant works of many authors (Ya. Eliashberg, P. Rabinowitz, C. Conley and E. Zehnder, M. Chaperon, J.-C. Sikorav, F. Laudenbach, Yu. Chekanov, A. Floer, H. Hofer, C. Viterbo, A. Weinstein, D. Salomon, A. Givental, M. Gromov, and others). Quantum and Floer cohomologies and Gromov's pseudoholomorphic curves theories are well-known byproducts of this development.

The Lagrange intersection theory is a far-reaching extension of the Morse theory, replacing the functions by such genuinely multivalued function as $\sqrt{x}$. Another extension replaces the functions by the closed 1-forms and Morse theory by Novikov's 
complex. The corresponding version of the Lagrange submanifolds intersection theory is due to J.-C. Sikorav.

I have heard that my initial conjectures, which triggered all these theories, are now proved (by Fukaya, Ono, Salomon, Ruan and others). Unfortunately I was unable to understand the technical details of these proofs. Kontsevich was unable to explain these details to me, while all the proofs are based on his theory of stable mappings of curves.

As far as I understand, all these proofs minorate the number of fixed points by the sums of the Betti numbers while I conjectured that it is minorated by the Morse number (or by the minimal number of geometrically different critical points if the fixed points are counted geometrically).

In my dreams of the 60 s the symplectomorphisms fixed points number minoration was followed by the similar study of the symplectic correspondences, which are not graphs of symplectomorphisms.

My idea of symplectic and contact topology was from the very beginning different from that of Gromov and Eliashberg, who were the first to explore the new domains discovered by the symplectization and contactization methods.

In their opinion, the symplectic (or contact) topology objects should have the "symplectic (or contact) homeomorphism invariance" property : they should persist under the $C^{0}$-small symplectic (or contact) diffeomorphisms.

To me the word "topology" with any adjective is the study of the discrete invariants of the continuous objects of the corresponding branch of geometry, be they homeomorphism invariant or not.

Thus, I include into projective topology the Möbius theorem on the three inflection points of a projective line deformations (claiming that a noncontractible circle, embedded generically into the real projective plane, has at least three inflection points, the dual curve having at least three cusps).

A recent conjecture in projective topology (due to F. Aicardi and D. Panov) extends the Möbius theorem to the generic surfaces in $\mathbb{R} P^{3}$, smoothly close to a plane. The conjecture claims, that the parabolic line of such a surface is tangent to the asymptotic direction of the surface at least at 6 points (generating 6 swallowtails of the dual surface) and that there are at least 4 parabolic lines if there are only 6 tangency points.

The recent spectacular achievements of contact topology include the creation by Yu. Chekanov (and also by Ya. Eliashberg and H. Hofer) of the contact homology theory. One may hope that the further development of these ideas would provide the proof of the conjectures on the necessity of four cusps of any wave front eversion in the plane.

This conjecture extends to the genuine multivalued function (of $\sqrt{x}$ type) the Sturm theorem on periodic functions, which itself extends the Morse theory of crit- 
ical points to higher derivatives. This theorem claims that the number of zeros of a function on a circle is minorated by the number of zeros of its first non vanishing Fourier harmonic (providing a confirmation of the general topological economy principle of algebraic objects).

The relation of all this domain to cyclic homology and characteristic classes, discovered by M. Kazarian, provides some hope that Morse theory can be extended to the higher derivatives (or to the more general case of nonholonomic constraints).

This extension should contain the (genuine) multivalued functions Morse theory - the theory of Lagrange intersections and Legendre linking (describing the causality in the relativistic physics, according to R. Low and R. Penrose) as the first derivative case.

Symplectic and contact topologies, created as the symplectization and the contactization of differential topology, are today well settled domains of mathematics.

Another attempt to multiply mathematical results is the complexification and quaternionization dream. The first spectacular success of this idea was the proof the Gudkov's conjecture in real algebraic geometry.

One of the most fundamental problems of mathematics is the problem of the topological structures of the real curves ${ }^{2}$ defined by algebraic equations of degree $n$.

This problem has been solved for $n=2$ by the Ancients (ellipses, hyperbolas, parabolas ... ). The cases $n=3$ or 4 were settled by Descartes and Newton. Hilbert has included the case $n=6$ in his 16 th problem. For $n=8$, the answer is still unknown.

These real problems are too difficult for the modern algebraic geometers and for present day computers.

According to Harnack, the number of connected components of a curve of degree $n$ in the real projective plane does not exceed $g+1$, where $g=(n-1)(n-2) / 2$ is the genus of the curve. Curves with $g+1$ components do exist, they were called $M$-curves ( $M$ for "maximal") by I.G. Petrovsky.

Hilbert announced that he had proved that only two arrangements of the 11 ovals of an $M$-curve of degree 6 were possible : only one oval contains other ovals inside its disc, the number of the interior ovals being either 1 or 9 .

A Nizhni Novgorod mathematician, D. Gudkov, submitted in 1970 his Thesis, proving that Hilbert was wrong. There exists one (and only one) more possible arrangement, for which the number of the interior ovals is 5. I.G. Petrovsky asked me to check this paper, contradicting both the Hilbert's statement and the preceding paper by Gudkov, in which he was proving the (wrong) Hilbert's claim.

\footnotetext{
${ }^{2}$ The Russian way to formulate problems is to mention the first nontrivial case (in a way that no one would be able to simplify it). The French way is to formulate it in the most general form making impossible any further generalisation.
} 
Mistakes are an important and instructive part of mathematics, perhaps as important a part as the proofs. Proofs are to mathematics what spelling (or even calligraphy) is to poetry. Mathematical works do consist of proofs, just as poems do consist of characters.

Leibniz started his calculus studies from the formula $d(u v)=d u d v$. Cauchy, the $\epsilon-\delta$ inventor, proved in his calculus course the continuity of the limit of the (nonuniformly) convergent series of continued functions. Lagrange's mistake in the linear ODE's theory hampered the development of linear algebra and of Jordan's form theory. The story of the Poincaré conjecture started from his confusion of homotopy with homology.

His "New methods of celestial mechanics" are the by-product of the attempts to prove a wrong statement (the by-product - the creation of the Dynamical Systems theory - being by far more important than this wrong statement "solving" a prize problem). The Burnside celebrated theorem on the groups of order $p^{a} q^{b}$ was first formulated by him wrongly. Leray told me, that his works on the hyperbolic PDE's were motivated by the remarkable paper of Petrovsky, who wrongly used the triviality of the cotangent bundle of every sphere (the paper was later modernised by Atiyah, Bott and Gårding). Kolmogorov's initial definition of the entropy of a dynamical system was wrong, as well as the Pontriagin's and Rokhlin's calculations of homotopy groups of spheres. I would be able to provide dozens of more recent examples of mistakes in celebrated papers if I did not fear for my life. I shall only mention a wrong symplectic reduction theorem in the first edition of the "Mathematical Methods of Classical Mechanics" (appendix 5,B).

The mistake of Hilbert, discovered by Gudkov, has lead to the foundation of the modern real algebraic geometry. Trying to understand Gudkov's very complicated paper, I have observed that in all his examples of $M$-curves of degree $2 k$, bounding orientable surfaces $B$, the following "Gudkov congruence" for the Euler characteristics holded

$$
\chi(B)=k^{2} \bmod 8 .
$$

Knowing the importance of the congruences mod 8 in the topology of 4-manifolds, I have decided to replace the real surface $B$ by its complexification of real dimension four.

The problem arose : how to complexify a manifold with boundary? This is a standard difficulty : the complexification is an informal operation for which there are no axioms, we should try to guess.

After several attempts I came to the following conclusion. Consider the real manifold with boundary, defined by the inequality $f(x) \geq 0$, where $f$ is a real function. To complexify it, replace the inequality by the equality $f(x)=y^{2}$. We guess, that the complexification of the real surface $B$ with boundary $f=0$, is the two-fold covering of the complement in $\mathbb{C} P^{2}$ of the Riemannian surface $f=0$ (which complexifies the boundary curve), ramified along the Riemannian surface. 
Applying the 4-dimensional topology result to this 4-manifold, I was able to prove the Gudkov congruence mod 4 (Rokhlin later extended it to the mod 8 case).

From this moment on, the real algebraic geometry entered the modern mathematics. In a series of brilliant works by Kharlamov, Nikulin, Viro, Shustin, Polotovski, Khovanski, Orevkov and others, a substantial progress was achieved. The interrelations of this domain to the "geometry of the formulae" (that is to the toric varieties and Newton polyhedra theory), to the "fewnomials" of Kushnirenko-SevastianovKhovanski and even to mathematical logic have been discovered.

Applying these ideas, I once suggested to I.M. Gelfand that they might simplify the complicated formulae of representation theory and of the hypergeometric functions theory : one should express their coefficients in terms of the convex polyhedra geometry. Gelfand (with his collaborators) immediately used this suggestion, finding new brilliant applications. He has always stressed (quite correctly) that mathematicians never appreciate new ideas, only the last step to the summit of the mountain counts in this mountain-climbing ${ }^{3}$

I shall now list some complexifications and quaternionizations of different mathematical objects. The trinities (real version, complex version, quaternionic versions) are listed below as the lines of a big informal commutative diagram, whose verticals are (mostly nontrivial) operations, transforming one trinity into another. Some of these operations are described in the comments below.

\footnotetext{
${ }^{3}$ M.M. Postnikov formulated an even more radical statement : the science never accepts new ideas, it fights against them. Most scientists at any given moment are working on horse-shoes and naturally do react negatively against the limousines ideas. See the curious attempt of S.-T. Yau to fight against Givental's theory in the present volume.
} 
(1)

\section{$\mathbb{R}$}

Morse theory anses attachment

$$
\begin{gathered}
\pi_{0}(\mathbb{R} \backslash 0)=\mathbb{Z}_{2} \\
\mathbb{R} P^{n}
\end{gathered}
$$

$\mathbb{R} P^{1}=S^{1}$

$\mathbb{R} P^{1} / \mathrm{Aut} \mathbb{R}=S^{1}$

Quadratic forms

Von Neuman - Wigner eigenvalues repulsion Möbius $S^{0}$ bundle

$$
S^{1} \rightarrow S^{1}
$$

Monodromy of a covering

$w$

$$
O, S O
$$

Tetrahedron

$$
\begin{gathered}
(4,4,6) \\
x^{2}+y^{3}+z^{4} \\
x^{3}+y^{3}+z^{3} \\
(\pi / 3, \pi / 3, \pi / 3) \\
A_{3} \multimap \multimap \\
2(1+3+3+5)=24 \\
(2,4,4,6) \\
D_{4} \sum_{0} \circ \\
E_{6} \circ 0 \\
\mathbb{C}[t]
\end{gathered}
$$

Numbers

H
$\mathbb{C}$

Picard-Lefschetz theory

Dehn twist

$\pi_{1}(\mathbb{C} \backslash 0)=\mathbb{Z}$

$\mathbb{C} P^{n}$

$$
\mathbb{C} P^{1}=S^{2}
$$

$\mathbb{C} P^{2} /$ Aut $\mathbb{C}=S^{4}$

Hermitian forms

Quantum Hall effect and Berry phase Hopf $S^{1}$ bundle

$$
S^{3} \rightarrow S^{2}
$$

Curvature of a connection

$c$

$$
U, S U
$$

Octahedron

$$
(6,8,12)
$$

$x^{2}+y^{3}+y z^{3}$

$x^{2}+y^{4}+z^{4}$

$(\pi / 2, \pi / 4, \pi / 4)$

$B_{3} \Longleftarrow 0$

$2(1+5+7+11)=48$

$(2,6,8,12)$

$$
\begin{aligned}
& F_{4} \curvearrowleft œ \\
& E_{7} \circ \cdots \\
& \mathbb{C}\left[t, t^{-1}\right]
\end{aligned}
$$

Trigonometric numbers

K
$\mathbb{H}$

$$
?
$$$$
\pi_{3}(\mathbb{H} \backslash 0)=\mathbb{Z} ?
$$

$\mathbb{H} P^{n}$

$$
\mathbb{H} P^{1}=S^{4}
$$

$\frac{\mathbb{H} P^{4} / \mathrm{Aut} \mathbb{H}}{\operatorname{Conj}}=S^{13}$

Hyperhermitian forms

$?$

Hopf $S^{3}$ bundle

$$
S^{7} \rightarrow S^{4}
$$

Hypercurvature of a hyperconnection?

$$
p
$$

Sp, ?

Icosahedron

$(12,20,30)$

$x^{2}+y^{3}+z^{5}$

$x^{2}+y^{3}+z^{6}$

$(\pi / 2, \pi / 3, \pi / 6)$

$$
\mathrm{H}_{3} \stackrel{5}{\circ} \mathrm{\circ}
$$

$2(1+11+19+29)=120$

$(2,12,20,30)$

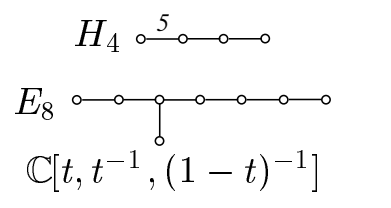

Elliptic numbers

Ell

Few comments to this self-explanatory table follow.

$(2) \rightarrow(3)$ : Morse theory describes the modification of the real level hypersurface 
of a smooth function, due to the jump of the noncritical value from one of the two connected components of the noncritical values set to the other. Picard-Lefschetz theory describes the Dehn twist of the complex level hypersurface of an holomorphic function, due to the motion of the noncritical value around the critical one.

$(5) \rightarrow(6):$ I have used the diffeomorphism $\mathbb{C} P^{2} /$ Conj $\approx S^{4}$ in my 1971 paper on real algebraic geometry as a well known fact. But Rokhlin told me that the proof of this fact, known to Pontriagin in the $30 \mathrm{~s}$, had never been published. I therefore asked experts (including Kuiper, who was visiting Moscow - which I was unable to leave - for the IMU buisness) whether they were aware of this fact, and later Kuiper and Massey published their proofs.

My original (1971) proof, based on the theory of the hyperbolic PDE's, provides also the real and the quaternionic versions of the Pontriagin's theorem : the proofs of the three versions are identical.

$(6) \rightarrow(7)$ : Hermitian (hyperhermitian) quadratic forms in complex (quaternionic) vector spaces are real quadratic forms, invariant under the action of the multiplication of the vectors by complex numbers (quaternions) of norm 1 . This definition is missing in the algebra textbooks.

The proof of (6) is based on the hyperbolicity of the cone of the degenerated real quadratic forms. The cones of the degenerated hermitian (hyperhermitian) forms are also hyperbolic, providing such generalisations of (6), as

$$
S^{11} / U(2)=\mathbb{C} P^{5} / S U(2)=\mathbb{H} P^{2} / S^{1}=S^{7}, \quad S^{23} / S p(2)=S^{13} .
$$

The three hyperbolic cones (corresponding to the real quadratic, Hermitian and hyperhermitian forms) are in fact universal varieties, providing the "Schur index" of representation theory.

$(7) \rightarrow(8)$ : The Wigner-von Newman theorem of the eigenvalues repulsion is based on the fact that the codimension of the variety of quadratic forms with multiple eigenvalues (in the space of the quadratic forms in an Euclidean space) is equal to 2.

In my 1972 paper "Modes and quasimodes" I have studied the monodromy of the eigenvectors fibration over the complement of this codimension two variety as well as the Hermitian case (where the codimension of the variety of the forms having a multiple eigenvalue is equal to 3 ).

As S.P. Novikow later explained to me, the complexification of this study implies the topological theory of the (integer) quantum Hall effect. I had also missed in 1972 the Berry phase theory, describing the natural adiabatic connection of the eigenvectors fibration in the Hermitian case.

The present situation of the hyperhermitian case is in a sense similar to that of the Hermitian case in 1972. The mathematical theory is ready, but its physical applications are still to be named. These applications should be interesting : the 
complexification of the monodromy being the connection notion, its quaternionization (which is also the complexification of the connection ) should provide a 4-form, measuring the dependence of the complex connection from the complex structure.

$(8) \rightarrow(9)$ : The restriction of the eigenvectors bundle to the link $S^{1}$ of the variety of the quadratic forms having a multiple eigenvalue (at its regular point) is the Möbius bundle (sending the boundary of the Möbius band to its central circle). In the Hermitian (hyperhermitian) case the transversal to the multiple eigenvalues variety forms is $\mathbb{R}^{3}$ (respectively $\mathbb{R}^{5}$ ), therefore the link in $S^{2}$ (respectively $S^{4}$ ). The eigenvectors bundle's restriction to this link is the Hopf bundle.

$(9) \rightarrow(10)$ : The "hypercurvature" should be a 4-form, providing the first Pontriagin class. Its geometrical description as of the complexification of the curvature might hopefully provide more topological information than its probable relation to the square of the curvature form and to the Chern-Simons theory.

The algebra of forms, generated by the Chern forms of the eigenvectors bundles, has been studied recently by B. Shapiro and his collaborators; they have also proved the degeneration of the spectral sequence, corresponding to the stratification of the Hermitian forms space accordingly to the eigenvalues multiplicities.

The real and hyperhermitian cases seems to be unsettled in these theories.

$(1) \rightarrow(13)$ : It is difficult to believe that the octahedron is the complex version of the tetrahedron, and icosahedron - of the octahedron. I first deduced it from the lecture of D. Kazhdan at the 80th Gelfand's birthday celebration at Rutgers (1993). The strange numerology below provides some confirmation of the mysterious parallelisms of all mathematical trinities, such as the parallelisms $(1) \rightarrow(13)$ or $(1) \rightarrow(22)$.

$(13) \rightarrow(14):$ The numbers of edges have the following property :

$$
6=2.3, \quad 12=3.4, \quad 30=5.6 .
$$

We recognise in the trinity $(2,3,5)$ the codimensions of the varieties of the multiple eigenvalues quadratic, Hermitian and hyperhermitian forms. Of course, we have also

$$
2=1+\operatorname{dim} \mathbb{R}, \quad 3=1+\operatorname{dim} \mathbb{C}, \quad 5=1+\operatorname{dim} \mathbb{H}
$$

and the numbers

$$
3=2+\operatorname{dim} \mathbb{R}, \quad 4=2+\operatorname{dim} \mathbb{C}, \quad 6=1+\operatorname{dim} \mathbb{H}
$$

are the codimensions of the varieties of the degenerated forms.

$(13) \rightarrow(15)$ : The rotation group $\Gamma \subset S O(3)$ of a regular polyhedron (13) is covered twice by the "binary group" $\widetilde{\Gamma} \subset \operatorname{Spin}(3)=S U(2)$, acting on $\mathbb{C}^{2}$. Its orbit space is the surface of zeros of the polynomial (15). 
$(15) \rightarrow(16)$ : The simple singularities (15) are "fenced" from the sea of the nonsimple ones by the "parabolic" (or "affine $E_{6}, E_{7}, E_{8}^{\prime \prime}$ ) singularities, of which the polynomials (16) are the simplest representatives.

$(16) \rightarrow(17)$ : Consider a triangle which one can buy in a stationery, (17). The affine reflection group defined by the triangle acts on the corresponding elliptic curve. The fencing singularity (16) can be obtained from this action on the elliptic curve essentially by the same invariant theory construction which builds the simple singularity (15) from the action of the binary group of a regular polyhedron (13) on the rational curve $\mathbb{C} P^{1}$.

$(13) \rightarrow(18):$ The symmetry groups (18) of regular polyhedra (13) are generated by reflections.

$(18) \rightarrow(19):$ The mirrors of a reflection group (18) of order $|W|$ subdivide the 3 -space into $|W|$ simplicial cones, called the Weyl chambers.

The three boundary mirrors of a chamber subdivide the 3-space into 8 larger pyramids - the Springer cones. The summands in (19) represent the numbers of the Weyl chambers in each Springer cone. Note the prime summands.

$(19) \rightarrow(20) \rightarrow(21) \rightarrow(13):$ Adding one to the numbers of line (19) one gets the degrees (20) of the invariants of the reflection groups (21) and also the numbers of vertices, faces and edges of the polyhedra (13).

$(22) \rightarrow(23)$ : The classical "folding", transforming $E_{8}$ into $H_{4}$, transforms also $E_{7}$ into $F_{4}$ and $E_{6}$ into $D_{4}$ (generated by $e_{1}-e_{2}, e_{2}-e_{3}, e_{3}-e_{4}$ and $2 e_{1}$ instead of $\left.e_{1}+e_{2}\right)$.

$(15) \rightarrow(22):$ The monodromy groups of the simple singularities (15) are the Euclidean reflection groups (22).

$(13) \rightarrow(22)$ : The J. MacKay correspondence describes the Dynkin diagrams of the (extended) root systems (22) directly in terms of the representation theory of the binary group of the corresponding regular polyhedron (13).

$(11) \rightarrow(23)$ : Gabrielov observed that in the polylogarithmic formulae for the characteristic classes studied in his works with Gelfand, Losik and Mc Pherson, the two poles case corresponded to the Chern class and the three poles case to the Pontriagin class.

$(23) \rightarrow(24):$ The Turaev-Frenkel theory of the elliptic numbers relation to modular hypergeometric functions is described in their paper in the "Arnold-Gelfand Mathematical Seminars", Birkhäuser 1997.

$(23) \rightarrow(25)$ : The trinity, consisting of cohomology, K-theory and elliptic theory, has been suggested by A.B. Givental.

It is interesting that the complexification of a manifold is by no way unique, depending on the structures we are interested in. Thus, the complexification of 
$S^{1}=\mathbb{R} P^{1}$ being $\mathbb{C} P^{1}=S^{2}$ that of $S^{1}=S O(2)$ is $S U(2)=S^{3}$. This is nice, making it possible to complexify the Möbius bundle

$$
S^{0} \rightarrow\left(S^{1}=S O(2)\right) \rightarrow\left(S^{1}=\mathbb{R} P^{1}\right)
$$

to obtain the Hopf bundle

$$
S^{1} \rightarrow\left(S^{3}=S U(2)\right) \rightarrow\left(S^{2}=\mathbb{C} P^{1}\right) .
$$

Trinity (6) suggests that the complexification of $S^{1}$ is sometimes also $S^{4}$.

Trinity (3) suggests that the complexification of $\mathbb{Z}_{2}$ is $\mathbb{Z}$. This is confirmed also by the following construction (originating from one of the attempts to complexify the braid groups and to quaternionize the permutation groups).

Consider the set $E$ of homotopy classes at mappings from a Lie group $G$ to itself preserving the identity. Define the (virtually noncommutative) "addition" in $E$ by $(f+g)(x)=f(x) g(x)$ and the "multiplication" by $(f g)(x)=f(g(x))$. The resulting algebraic structure on $E$ seems to have no name (the distributivity holding only from one side), so I shall call this "ring" $E$ the ellipse of $G$.

The ellipse of $S^{0}$ is the ring $\mathbb{Z}_{2}$ of two elements, that of $S^{1}=S O(2)$ is the ring $\mathbb{Z}$. The ellipse of $S^{3}=S U(2)$ is also $\mathbb{Z}$ (I would prefer to get the Gauss numbers ring as the complexification of $\mathbb{Z}$ ).

To complexify $\mathbb{Z}$ one can also consider it as the died braid group $\operatorname{IBr}(2)$ where

$$
\operatorname{IBr}(n)=\pi_{1}\left(\mathbb{C}^{n} \backslash \mathbb{C} \text {-mirrors of } A_{n}\right),
$$

or as the ordinary braid group $B r(2)$, where

$$
\operatorname{Br}(n)=\pi_{1}\left(\operatorname{Config}_{n} \mathbb{C}\right) .
$$

The died braid group $\operatorname{IBr}(n)$ is the complexification of the symmetric group:

$$
\pi_{0}\left(\mathbb{R}^{n} \backslash \mathbb{R} \text {-mirrors of } A_{n}\right) \approx S(n) .
$$

To complexify the fundamental group $\pi_{1}(X)$ one is tempted to write $\pi_{1}(X)=$ $\pi_{0}(\Omega X)$ and to complexify it to $\pi_{1}\left({ }^{\mathbb{C}} \Omega{ }^{\mathbb{C}} X\right)$. The complexification of the loop space is perhaps related to the holomorphic loops. Applying these ideas to $X=\mathbb{C} \backslash 0$, we should consider as complex loops the polynomial mappings of $\mathbb{C}$ into $\mathbb{C}^{2} \backslash 0$ (the boundary conditions at infinity being provided by the highest degree terms).

The Zariski theorem reduces the calculation of the fundamental group of the space of these pairs of polynomials to the calculation of the fundamental group of the complement of a single rational curve in $\mathbb{C}^{2}$ (which is the image of a generic polynomial mapping of $\mathbb{C}$ of a given degree). I guess that the final answer is either $\mathbb{Z}$ 
or $\mathbb{Z}^{2}$, depending on whether the boundary conditions at infinity correspond to the complexification of the died braid group $\mathbb{Z} \approx \operatorname{IBr}(2)$ or to the ordinary braid group $\operatorname{Br}(2) \approx \mathbb{Z}$.

The quaternionic versions of the Coxeter and Shephard-Todd groups are still to be defined and classified. These versions might be Lie groups whose orbit spaces are smooth, or groups generated by the "quaternionic reflections". One might speculate that the existence of the sporadic simple groups is related to what remains from the real and complex reflection groups in the quaternionic version of the reflection groups trinity.

The complexification of the orientation is of course a nowhere vanishing holomorphic highest degree form. B. Khesin and I. Frenkel have suggested that the complexification of the cohomology theory should be the Leray theory of meromorphic differential forms. Using this theory, Khesin and Rosly were able to define the "complex linking number" of two complex curves in some complex 3-manifolds (generalising the Gauss integral formula for the linking number of real curves in the real 3 -space).

In mathematics we always encounter mysterious analogies, and our trinities represent only a small part of these miracles. I might mention, as an example, the "strange duality" of the Lobachevsky triangles, which I discovered in 1974 and which is now explained by V. Batyrev as the first manifestation of the general mirror symmetry of physicists. As an example of a still puzzling mystery, I shall mention the $R$. Faure's duality, relating the particle moving with energy $E$ in the field with potential $U(z)=|d w / d z|^{2}$ with the particle moving with energy $-1 / E$ in the field with potential energy $V(w)=-|d z / d w|^{2}$, whatever be the holomorphic function $w(z)$. The function $w=z^{2}$ provides the classical duality between the Hooke and gravitation or Coulomb forces, but the above duality is quite general and holds both in the classical and in the quantum version.

A commented list of several hundred of problems, originating from such "experimental facts", is prepared by my Moscow seminar participants and will hopefully be published soon.

The attempts to complexify and to quaternionize mathematical theories are making clear the fundamental unity of all parts of mathematics. The growing specialisation and the bureaucratic subdivision of mathematics into small domains becomes an obstacle to its development. The organisers of the International Congress of Mathematicians in Berlin in 1998 have considered their bureaucratic sections as scientifically independent unities. As a result, parallel talks, formally belonging to different sections, were in fact devoted to the same subject, be it called symplectic geometry, mathematical physics, differential topology, partial differential equations, global analysis, quantum mathematics or infinite dimensional Lie algebra theory. 
Several invited speakers of the Berlin Congress told me that they would rather have listened to each other's talk, but were unable to do it, speaking simultaneously.

This lack of understanding of the interrelations between different domains of mathematics originates from the disastrous divorce of mathematics from physics in the middle of the 20th century, and from the resulting degeometrisation of the mathematical education.

Criticising my statement that mathematics is a part of physics, one of the former best Bourbaki leaders ${ }^{4}$ has written to me in June 1998 : "Mathematics is completly different from physics ... Mathematicians should not write on such philosophical questions, since even the best of them can write pure nonsense".

It is interesting to compare this boomerang statements with the words of Hilbert, who wrote in 1930 : "Geometry is nothing more than a branch of physics; the geometrical truths are not essentially different from the physical ones in any aspect and are established in the same way" (Naturerkennen und Logik, Naturwissenschaften, 1930, 959-963).

Hilbert tried to predict the future development of mathematics and to influence it by his Problems. The development of mathematics in the 20th century has followed a different path. The most important achievements - the flourishing of homotopy theory and of differential topology, the geometrisation of all branches of mathematics, its fusion with the theoretical physics, the discovery of the algorithmically undecidable problems and the appearance of computers - all this went in a different (if not opposite) direction.

The influence of $\mathrm{H}$. Poincaré and of $\mathrm{H}$. Weyl on the science of the 20th century was much deeper. To Poincaré, who created modern mathematics, topology and dynamical systems theory, the future of mathematics lay in the development of mathematical physics, oriented to the description of the relativistic and quantum phenomena. Among other important things, Poincaré explained, that only non interesting problems might be formulated unambiguously and solved completely. According to Poincaré, one should rather try to understand, what may be changed in the problem formulation. He had in mind first of all the variation of the coefficients of the equations in bifurcation type problems and all kinds of general position arguments - the topics which are now called singularity theory, global analysis and functional analysis. Interestingly enough, what is now called versal deformation theorem had already been proved in his Thesis (for the case of zero dimensional holomorphic complete intersections) as lemma 4, basis for his bifurcation theory.

The mathematics of the 20th century mostly followed the road shown by Poincare (the main difficulty being - as A. Weil once told me - the fact that too many good

\footnotetext{
${ }^{4}$ who declined the invitation to participate in the present book, explaining that, according to his experience, all collective works are failures.
} 
mathematicians have appeared, whereas all valuable mathematicians knew personnally each other at Poincaré's time).

According to Kolmogorov, Hilbert was seriously worried by what would happen to the Mathematische Annalen cover in 500 years : he thought that the names of the former Editors would fill up all the space.

Kolmogorov objected to Hilbert that our culture would probably not survive for such a long period : the united bureaucrats of all countries will soon be able to stop all kind of creativity, making further mathematical discoveries impossible, as are geographical discoveries today.

We can imagine that some of the most appealing domains of mathematics will be transformed into wilderness preserves, where rich people will be able to buy for an expensive price the pleasure to hunt one-two theorems, guided by the scientific jaegermeisters.

It is difficult to decide, which of these predictions is more likely to happen. It seems however rather clear that the centre of the humanity will soon move from wealthy Europe and North America to hungry Asia, where our culture has few chances to survive.

I would like to hope that this prediction is as wrong as the others. Discussing these perspectives, the optimist $\mathrm{H}$. Whitney insisted that America is still producing excellent mathematicians in spite of the sad fact that its general cultural and educational level is already almost as low as that of the future global attractor.

One may also hope that the coming nuclear civil wars and military confrontations will lead to a better appreciation of science by society and to a paradoxical flourishing of world mathematics (similar to the flourishing which occurred in Russia after the awful Bolshevik revolution).

CEREMADE, UMR CNRS N ${ }^{\circ} 7534$

Université Paris-Dauphine

Place du Maréchal de Lattre de Tas-

signy

75775 PARIS CEDEX 16 (FRANCE)

Steklov Mathematical Institute

8, Gubkina Street

117966 MOSCOW, GSP-1, (RUSSIA) 